\title{
The opioid peptide biphalin modulates human corneal epithelial wound healing in vitro
}

\section{Ozgun Melike Gedar Totuk ( $\square$ melikegedar@gmail.com )}

Bahcesehir University https://orcid.org/0000-0003-1863-6501

\section{Erdost YILDIZ}

Koc Universitesi

\section{Adriano MOLLICA}

Universita degli Studi Gabriele d'Annunzio Chieti e Pescara Dipartimento di Farmacia

Kerem KABADAYI

Bahcesehir Universitesi Tip Fakultesi

\section{Afsun SAHIN}

Koc Universitesi

\section{Research article}

Keywords: synthetic opioid peptide, corneal wound healing, opioid receptors, human corneal epithelial cell culture

Posted Date: March 31st, 2020

DOI: https://doi.org/10.21203/rs.2.11471/v2

License: (c) (i) This work is licensed under a Creative Commons Attribution 4.0 International License. Read Full License 


\section{Abstract}

Background: Analgesic drugs, including nonselective opioids and non-steroidal anti-inflammatory drugs, should be used with great precautions to relieve pain after physical damage of the corneal epithelium because of their unfavorable effects on the wound-healing process. Biphalin is a synthetic opioid peptide that has been demonstrated to possesses a strong analgesic effect on rodents. The purpose of this study is to investigate the effects of biphalin on human corneal epithelium wound healing.

Methods: An immortalized human corneal epithelial cell (HCEC) culture was used to test the effects of biphalin on wound healing. The toxicity of biphalin in various concentrations was measured with the MTT assay. The effect of $1 \mu \mathrm{M}$ and $10 \mu \mathrm{M}$ biphalin were tested on wound closure in an in vitro scratch assay of HCECs and for cell migration and proliferation separately. Naloxone, a non-selective competitive antagonist of opioid receptors, was also used to inhibit the effects of biphalin in all experiments.

Results: Biphalin did not cause any toxic effect on HCECs in concentrations lower than $100 \mu \mathrm{M}$ at various incubation time points. Biphalin increased the wound closure process significantly at $1 \mu \mathrm{M}$ concentration in an in vitro scratch assay of HCECs $(p<0.05)$. It also increased the migration of HCECs significantly ( $p$ $<0.01$ ). There was no significant difference between biphalin and control groups of HCECs in the Ki67 proliferation assay.

Conclusion: Biphalin, a synthetic opioid peptide, has a potential role as a novel topical analgesic agent that promotes corneal epithelial wound healing. This role should be evaluated in further in vivo and clinical studies.

\section{Background}

Ocular pain is an important symptom of inflammatory or traumatic disorders affecting anterior segment structures, such as the cornea, sclera, conjunctiva, and uvea. It is commonly relieved with ophthalmic topical anesthetic agents, such as tetracaine, procaine, benoxinate, and proxymetacaine (proparacaine), most of which have toxic side effects to the corneal epithelium, such as corneal epithelial erosion and delayed wound healing. [1, 2]

Opioids are considered potent and appropriate analgesics for moderate to severe acute and chronic pain and have been used to treat pain since early ages. They are used in ophthalmology by a systemic or periocular (sub-Tenon's block, peribulbar, retrobulbar) administration route. [3, 4] In addition to their analgesic effect, opioids show cytoprotective, neuroendocrine regulatory, immunomodulatory, and behavioral-modification effects by affecting opioid receptors. [5] Opioids interact with three receptor classes of seven heterotrimeric inhibitory transmembrane G-protein-coupled opioid receptors (GCPRs): delta opioid (DOR), kappa-opioid (KOR), and mu-opioid (MOR). [6]

Biphalin is a dimeric nonspecific opioid analog [(Tyr-D-Ala-Gly-Phe-NH-)2], which mainly activates DORs and MORs, resulting in a proven complete analgesic response and neuroprotective effect in the central 
nervous system. [7] Biphalin exerts less dependence and tolerance compared to morphine, since DORand MOR-induced side effects are believed to be prevented by activation of KOR. [8] To date, biphalin has been shown to be one of the most potent peptide-based opioid analgesics and has been recently investigated as a potential treatment for abdominal pain associated with inflammatory bowel disease. [9] The analgesic potency of biphalin is seven times greater than that of etorphine and three orders of magnitude greater than morphine after intracerebroventricular administration. [10] Since common analgesic drugs used to relieve pain after physical damage to the corneal epithelium have unfavorable effects on the wound-healing process, the search for an analgesic that has no adverse effect on corneal wound healing continues.

To the best of our knowledge, the role of synthetic opioid peptides on human corneal epithelial cells has not been investigated in the published English literature. Since biphalin is a nonspecific opioid analog, this study aimed to evaluate the effects of biphalin on human corneal epithelial cells, particularly on wound healing, cell migration, and cell proliferation in vitro.

\section{Methods}

\section{Synthesis of the Opioid Agonist}

Biphalin was synthesized by Adriano Mollica at his laboratory in Università degli Studi G. d'Annunzio Chieti e Pescara, Department of Pharmacy, Chieti, Italy. The peptide's chemical properties were in full agreement with those already reported in the literature (Fig. 1). [11]

\section{Corneal Epithelial Cell Culture}

Human immortalized corneal epithelial cells (HCECs) were a generous gift from Dr. James Jester (Irvine, CA, USA). The HCECs were cultured in a keratinocyte serum-free medium (KSFM; Gibco, NY, USA) supplemented with bovine pituitary extract (BPE; $25 \mu \mathrm{g} / \mathrm{mL}$ ), epidermal growth factor (EGF; $50 \mathrm{ng} / \mathrm{mL}$ ), penicillin $(100 \mathrm{IU} / \mathrm{mL})$, and streptomycin $(100 \mu \mathrm{g} / \mathrm{mL})$. The cells were maintained in $75 \mathrm{~cm}^{2}$ flasks until experimentation. The HCECs did not differentiate in the keratinocyte serum-free medium. To differentiate HCECs, we followed the protocol described by Sahin et al. [12] We changed the medium with DMEM with $10 \%$ FBS. In this way, the HCECs were differentiated and stratified.

\section{Cytotoxicity Assay}

The HCECs were treated with different concentrations of biphalin (from $1 \mathrm{pM}$ to $100 \mu \mathrm{M}$ ) in 96-well culture dishes (Corning, NY, USA) for 24 hours. The cytotoxicity of exposure was measured with the MTT (3-(4,5-dimethylthiazolyl-2)-2, 5-diphenyltetrazolium bromide) assay (Thermo Fisher Scientific, MA, USA). The color of the MTT tetrazole salt was measured with a spectrophotometer at a wavelength of $570 \mathrm{~nm}$. 


\section{In Vitro Scratch Assay}

The HCECs were grown to confluence on 12-well culture dishes (Corning, NY, USA). On reaching confluence, the cells were rinsed with a phosphate-buffered saline solution (PBS) and exposed to a differentiation medium consisting of Dulbecco's Modified Eagle Medium (DMEM; Gibco, NY, USA) with $10 \%$ fetal bovine serum (FBS), penicillin $(100 \mathrm{IJ} / \mathrm{mL})$, and streptomycin $(100 \mu \mathrm{g} / \mathrm{mL})$ for one day. Two perpendicular linear scratches were made using a sterile $200 \mu \mathrm{L}$ pipette tip, and the wells were washed with PBS. Immediately after the scratch, all groups were incubated in KSFM. Biphalin (in two different concentrations; $1 \mu \mathrm{M}\left[10^{-6} \mathrm{M}\right]$ and $10 \mu \mathrm{M}\left[10^{-5} \mathrm{M}\right]$ ) or biphalin plus naloxone solution (in two different concentrations; $1 \mu \mathrm{M}$ and $10 \mu \mathrm{M}$ ) or their vehicle (PBS) was added to the cultures. Naloxone, which is a selective opioid receptor antagonist, was added to inhibit the opioid receptor-related effects of biphalin. The scratch area was captured hourly for 24 hours using live-cell microscopy (DMi 8; Leica, Wetzlar, Germany). The relative wound area (RWA) was measured using ImageJ software (National Institutes of Health [NIH], MD, USA) (Fig. 2).

\section{Transwell Migration Assay}

After wounding, the cells were used to do a Transwell Migration Assay. This is because during confluency, gene expressions of cells are different from the wound-healing situation. By creating a wound, we simulated the wound process in epithelial cells. Immediately after the wounding process was complete, as described above, the HCECs were trypsinized, washed, and plated $\left(2.5 \times 10^{5}\right.$ cells per insert $)$ in $8.0 \mu \mathrm{m}$ pore size Transwell inserts (Corning, NY, USA) in KSFM.

The lower compartment was filled with DMEM with $10 \%$ FBS or DMEM with $10 \%$ FBS plus either biphalin (in two different concentrations; $1 \mu \mathrm{M}$ and $10 \mu \mathrm{M}$ ) or biphalin plus naloxone solution (in two different concentrations; $1 \mu \mathrm{M}$ and $10 \mu \mathrm{M}$ ) or their vehicle (PBS). After 24 hours, the cells on the upper side of the insert were removed by scraping, and the cells that had migrated through were fixed on the lower side of the membrane with $4 \%$ paraformaldehyde, then stained with hematoxylin-eosin and quantified by counting the number of cells in 10 separate fields. The data were expressed as the number of migrated cells per micrograph field for each sample well.

\section{Ki67 Proliferation Assay}

The effect of biphalin on in vitro proliferation was assessed by immunofluorescence staining for Ki67. The Ki67 protein is present during the $\mathrm{G} 1, \mathrm{~S}, \mathrm{G} 2$, and $\mathrm{M}$ phases of the cell cycle and is strictly associated with cell proliferation. $2.5 \times 10^{5} \mathrm{HCEC}$ were plated in equal numbers in 24-well culture dishes (Corning, NY, USA). After reaching confluence, cells were rinsed twice with PBS and exposed to a stratification medium consisting of DMEM with $10 \%$ FBS. Two perpendicular linear scratches were made using a sterile $200 \mu \mathrm{L}$ pipette tip, and the wells were washed three times with PBS and incubated with KSFM 
without EGF and BPE. Immediately after the scratch, biphalin (in two different concentrations; $1 \mu \mathrm{M}$ and $10 \mu \mathrm{M}$ ) or biphalin plus naloxone solution (in two different concentrations; $1 \mu \mathrm{M}$ and $10 \mu \mathrm{M}$ ) or their vehicle (PBS) were added to the cell culture medium. The cells were incubated for 6 hours at $37^{\circ} \mathrm{C}$. After the treatment, the cells grown on 24-well culture dishes were fixed in 4\% paraformaldehyde for 20 min. After three washes with PBS, the cells were incubated with $0.1 \%$ TritonX-100 in PBS for 8 min. The cells were incubated with Superblock (Thermo Fisher Scientific, MA, USA) for $10 \mathrm{~min}$ at room temperature and then overnight at $4^{\circ} \mathrm{C}$ with the rabbit anti-Ki67 primary antibody (Abcam, MA, USA) at optimal dilutions in a blocking solution. After three washes with PBS, the cells were incubated with the FITC-conjugated secondary antibody (Abcam, MA, USA) for 90 min at $37^{\circ} \mathrm{C}$, then washed, counterstained with 406diamidino-2-phenylindole (DAPI), and mounted. Negative controls were stained in a similar fashion (DMi 8; Leica, Wetzlar, Germany). The Ki67 proliferation index is the proportion of Ki67 stained cell nuclei to DAPI stained cell nuclei from 20 different micrograph areas. The Ki67 proliferation index is calculated by dividing the number of Ki67 stained cell nuclei by the number of DAPI stained cell nuclei using ImageJ software (National Institutes of Health [NIH], MD, USA)

\section{Gene Expression Analysis of Opioid Receptors with Quantitative Reverse Transcription PCR}

The presence and expression levels of MOR, DOR, and KOR in the HCECs were measured quantitatively using a real-time polymerase chain reaction (qRT-PCR). The presence of OPRM1, OPRD1, and OPRK1 mRNAs, which are related with mu, delta, and kappa opioid receptor proteins, respectively, was studied in differentiated and undifferentiated HCECs and in SH-SY5Y cell lines. SH-SY5Y cell lines are human neuronal cancer cell lines that have demonstrated expressions of these three opioid receptors in previous literature.[13] To show the effect of the serum on HCECs, HCECs cultured in KSFM and in DMEM+10\% FBS were used in qRT-PCR analysis. The qRT-PCR primers for the genes that are responsible for the opioid receptor expressions selected is shown in Table 1. RNA isolation from cells was performed via a QuickRNA MicroPrep Kit (Zymo Research, Irvine, CA, USA). Extracted RNAs were quantified with Nanodrop 2000 spectrophotometry (Thermo Fisher Scientific, Waltham, MA, USA), and $1000 \mathrm{ng}$ cDNA was prepared using M-MLV Reverse Transcriptase (Invitrogen, Carlsbad, CA, USA). Quantitative real-time expressions of mRNAs were detected and compared with a Light Cycler 480 SYBR Green I Master (Roche, Basel, Switzerland).

\section{Statistical Analysis}

Each experiment was performed at least two times. For blind analysis, collection of images was made by E.Y., and each image was assigned a number. Then images were analyzed anonymously by K.K. Values were displayed as a mean \pm standard deviation. For the in vitro scratch assay, we measured timedependent change in the wound area. For other assays, we collected data only at one time point. Since two-way ANOVA is used to examine the interaction between two independent variables (treatment and 
time), and one-way ANOVA tests the effect of one independent variable (treatment), statistical analysis was performed using two-way ANOVA for in vitro scratch assay results, and one-way ANOVA with a Tukey's Honest Significant Difference test was used for other results to determine the degree of significance (R; R-Project, Vienna, Austria).

Results were considered statistically significant when the p-value was less than 0.05 .

\section{Results}

\section{MTT Toxicity Assay}

First, we measured the cytotoxic effect of biphalin on HCECs with the MTT toxicity assay. Biphalin has no cytotoxic effects at doses lower than $100 \mu \mathrm{M}$ after a 24-hour drug incubation (Fig. 3). Because of this, we selected two doses, $1 \mu \mathrm{M}$ and $10 \mu \mathrm{M}$, to apply on HCECs during in vitro experiments.

\section{The In Vitro Scratch Assay}

We observed a statistically significant decrease in RWA in biphalin-treated cells at $1 \mu \mathrm{M}$ concentration compared to the vehicle group (at the $6^{\text {th }}$ hour, biphalin RWA $=0.02126 \pm 0.02299$, biphalin plus naloxone $R W A=0.06015 \pm 0.06286$, vehicle RWA $=0.1552 \pm 0.16940, n=8 p<0.05$ ) (Fig. 4a). However, there was no statistically significant difference in RWA in $10 \mu \mathrm{M}$ biphalin, biphalin plus naloxone, or the vehicle groups (at the $6^{\text {th }}$ hour, biphalin RWA $=0.05838 \pm 0.06159$, biphalin plus naloxone RWA $=0.06015 \pm 0.06897$, vehicle RWA $=0.01507 \pm 0.02983, n=8)$ (Fig. $4 b$ ). It shows the positive wound-healing effect of biphalin in the in vitro wound-healing model of HCECs. But this effect could be because of proliferation or migration. To understand which cell behavior leads to the positive effect, we performed a Transwell Migration Assay and a Ki67 proliferation assay.

\section{Transwell Migration Assay}

We recorded a statistically significant increase $(n=20, p<0.01)$ in the number of cells passing through the Transwell membrane at $1 \mu \mathrm{M}$ concentration of biphalin (82.35 \pm 42.96$)$ in respect of vehicle (34.35 \pm 7.081$)$ and biphalin plus naloxone (52.75 \pm 23.76$)$ (Fig. 5a). However, there was no statistically significant difference between the $10 \mu \mathrm{M}$ concentration of biphalin ( $56.80 \pm 42.36)$, biphalin plus naloxone $(37.20 \pm 13.23)$, or the vehicle $(39.85 \pm 12.35)$ groups in terms of the number of cells passing through the membrane $(n=20)$ (Fig. 5b).

\section{Ki67 Proliferation Assay}

To examine the proliferative activity of HCECs during wound healing, Ki67 expression was observed in the wound areas $(n=20)$. After image acquisition, for a more precise comparison of Ki67 expression between 
the groups, the Ki67 labeling index was calculated. On the Ki67 proliferation index, there was no statistically significant difference between experimental groups in either concentration of biphalin, $1 \mu \mathrm{M}$ and $10 \mu \mathrm{M}$, at 3 and 6 hours (Fig. 6 ). These findings suggest biphalin has no significant proliferative effect on HCECs.

\section{Gene Expression Analysis with qRT-PCR of Opioid Receptors}

On qRT-PCR, we demonstrated the presence of OPRM1, OPRD1, and OPRK1 mRNAs in both differentiated and undifferentiated HCECs compared to the SH-SY5Y cell lines. MOR, DOR, and KOR mRNA expression was significantly lower $(p<0.001)$ in HCECs than in the SH-SY5Y cell lines (Fig. 7).

\section{Discussion}

In this study, we demonstrated that biphalin, which acts mainly via MORs and DORs, increases cell migration at the wound area and accelerates wound closure without affecting cell proliferation. Partial inhibition of the wound-healing effect of biphalin with naloxone can suggest MOR- and DOR-dependent mechanism of action. Our findings on biphalin are consistent with the wound-healing accelerator effects of MOR and DOR stimulations in cell cultures, which have been described previously. $[14,15]$ We also demonstrated that all three types of opioid receptors are present in HCECs using the qRT-PCR, which is also in line with previous reports.[16] To the best of our knowledge, this is the first study on the effects of a synthetic dimeric opioid peptide on human corneal epithelium cells in the literature.

Opioid receptors are distributed in the anterior segment tissues as well as in various layers of the retina and the optic nerve. They play important roles in the regulation of iris function, accommodation power, aqueous humor dynamics, corneal wound healing, retinal development, inhibition of neuroinflammation, neuroprotection against glaucomatous damage, and reduction of intraocular pressure. [17-20] Although various pharmacological mechanisms were proposed, exact processes underlying the effects induced by opioid receptors have not been clearly defined. Regarding opioid receptor types, both DOR and MOR were determined in the corneal tissues of certain animals. [21]

Limited data is available regarding the effects of opioids on corneal wound healing. Peyman et al. examined the analgesic and toxic effects of topical morphine on corneal abrasion in a rabbit corneal abrasion model. [22] They showed that morphine sulphate had a desirable analgesic property without irritating or causing any adverse effect on the cornea. Later, Stiles et al. studied the effect of topical application of a $1 \%$ morphine sulfate solution on signs of pain and wound healing in dogs with corneal ulcers and examined normal corneas immunohistochemically for the presence of MORs and DORs. [21] They showed that both MORs and DORs were present in normal canine corneas. They also demonstrated that topical morphine sulfate provided analgesia and did not interfere with normal wound healing. A recent study by Bigliardi et al. studied the effect of DORs in an in vitro scratch wound model, which we 
also used in our study. [14] They found that migration and wound recovery were enhanced in human keratinocyte monolayers overexpressing DORs in vitro. They concluded that opioid receptors affect intercellular adhesion and wound-healing mechanisms, underlining the importance of a neuroendocrine system in wound healing and homeostasis. In another study, Wang et al. showed the role of delta and mu opioid receptors on wound healing and migration in DOR and MOR knockout mice. [15]

Synthetic opioids have been known to mimic the cytoprotective effects of endogenous opioidergic ligands and induce their physiological effects by the activation of DORs, KORs, and MORs, which were further subclassified into delta1 and delta2; kappa1, kappa2, and kappa3; and mu1, mu2, and mu3 opioid receptors, respectively. [6] Biphalin (PubChem CID: 5487663) was first synthesized by Lipkowski et al. in 1982 and then resynthesized by Mollica et al. with a modification in which two identical enkephalin-like tetrapeptides (Tyr-DAla-GlyPhe) were connected "head to head" by a hydrazide bridge (Fig. 1). [11, 23] In this modification, the natural residues of the dimeric opioid peptide biphalin were replaced by the corresponding homo- $\beta 3$ amino acids. The derivative 1 containing $\mathrm{h} \beta 3$ Phe in place of Phe showed good MOR and DOR affinities and antinociceptive activity in vivo together with an increased enzymatic stability in human plasma. Biphalin has been shown to be one of the most potent peptide-based opioid analgesics. It crosses the blood-brain-barrier, resists enzymatic degradation, and exerts high metabolic stability in serum and the brain with half-lives of 87 and 193 min, respectively. [10] Biphalin exerts a higher binding affinity to DOR and MOR, greater analgesic potency than morphine and etorphine, and less dependence, excitatory hypersensitivity, and tolerance compared to morphine during chronic use. These DOR- and MOR-induced side effects of morphine are believed to be prevented by activation of KOR, and biphalin shows reduced MOR-related side effects due to the high potential of simultaneous MOR and DOR interactions and thus synergistic effects. $[7,8,24]$

Although the potential mechanisms of biphalin on promoting migration and accelerating wound healing in the corneal epithelium have not been evaluated in this study, inhibition of the influx of $\mathrm{Ca}+2$, inhibition of PKC-dependent $\mathrm{Na}-\mathrm{K}-\mathrm{Cl}$ cotransporter, activation of MAP kinases, and inhibition of reactive oxygen species production or DORs may play a role in the mechanisms of biphalin-induced effects. [19;25-27]

The antinociceptive effect of biphalin has been suggested to have a potential role in the treatment of cancer pain, abdominal pain associated with inflammatory bowel disease, and in neuropathic pain via different routes of administration, such as subcutaneous, intravenous, intrathecal, intracerebroventricular, and intraperitoneal. $[9,28,29]$ Furthermore, recent studies have revealed the beneficial role of biphalin on cell viability and neuroprotection against excitotoxic and ischemic damage by inhibiting protein kinase Cdependent sodium potassium-chloride cotransporter expression in focal brain ischemia by activating downstream survival mitogen-activated protein kinases, and by inhibiting reactive oxygen species production in an opioid receptor-dependent manner, which was challenged by naltrexone. $[19,25,30]$ Biphalin also acts as an immunomodulatory agent by stimulating human $\mathrm{T}$ cell proliferation, natural killer cell cytotoxicity in vitro, and interleukin-2 production and diminishes pro- and anti-inflammatory factors in lipopolysaccharide-treated microglial cells. [28,31] 
We proposed that the lack of a significant effect on proliferation of biphalin may be the result of its failure in binding to opioid growth factor receptors (OGFR), since OGFRs bear no resemblance to classical opioid receptors, and blockade of OGFR interaction with naltrexone accelerates cell growth. In the biphalin plus naloxone groups, both proliferation and wound healing were partially increased. We believe this effect was due to the binding of naloxone to OGFRs as a nonselective competitive opioid antagonist. [32]

In our study, there was no statistically significant difference between biphalin-treated cells at $10 \mu \mathrm{M}$ concentration and control groups on wound healing and cell migration. Biphalin shows approximately 100 times lower affinity to KOR than MOR and DOR. [33] Therefore, it is possible that at higher concentrations of biphalin, KORs are also activated and reverse the healing and migration process.

Many studies have demonstrated the cytoprotective and reformative effects of DORs and MORs separately, and their synergistic activation may have strengthened their positive effects. [34] In further experiments, the exact effects of specific opioid receptor subgroup activation on human corneal epithelial cells should be explored with specific opioid peptides. Additionally, the effect of biphalin on the woundhealing process in the cornea and changes in keratinocytes, neural fibers, dendritic cells, the basement membrane, and extracellular matrix elements and their interactions should also be investigated.

The main limitation of the present study was the lack of data on the effect of biphalin on the intracellular signaling mechanisms and cell metabolism. In future experiments, we plan to examine the effects of biphalin and its derivatives on corneal healing in experimental animals, which will allow us to determine receptor-down signaling pathway interactions. A second limitation of our study that must be addressed is the lack of an in vivo animal model. We plan to conduct studies in transgenic mice in order to shed light on the effects of opioids in corneal wound healing.

\section{Conclusion}

In the corneal epithelium, biphalin accelerates wound healing and cell migration without affecting cell proliferation. Since the analgesic and antinociceptive effects of biphalin have been demonstrated in previous studies, it can be used in the near future as an analgesic after corneal traumas and surgeries to modulate corneal epithelial wound healing. We also propose that biphalin may become a new treatment alternative not only for corneal traumas but also for other neuropathic pain syndromes affecting the ocular surface, such as herpetic or diabetic neuropathy.

\section{List Of Abbreviations}

bovine pituitary extract: BPE; delta opioid receptor: DOR; 406-diamidino-2-phenylindole: DAPI; 3-(4,5dimethylthiazolyl-2)-2, 5-diphenyltetrazolium bromide: MTT; Dulbecco modified eagle medium: DMEM; epidermal growth factor: EGF; fetal bovine serum: FBS; G-protein-coupled opioid receptors: GCPRs; human immortalized corneal epithelial cells: HCECs; kappa-opioid receptor: KOR; keratinocyte serum-free 
medium: KSFM; mu-opioid receptor: MOR; National Institutes of Health: NIH; phosphate-buffered saline solution: opioid growth factor receptors: OGFR; PBS; real-time polymerase chain reaction:qRT-PCR; relative wound area: RWA.

\section{Declarations}

Ethics approval and consent to participate: Since the study was a laboratory-based study not using patients or patient material, ethical approval was not required.

Consent for publication: Not applicable

Availability of data and material: Data sharing is not applicable to this article as no datasets were generated or analyzed during the current study.

Competing interests: The authors declare that they have no competing interests.

Funding: The design of the study and collection, analysis, and interpretation of data and in writing the manuscript was not funded.

Author Contributions: All authors have made substantial contributions to all of the following: (1) the conception and design of the study: OMGT, AS, EY (2) Performed the experiments: EY, KK (3) Analysis and interpretation of data: EY, KK (4) Contributed reagents/materials/analysis tools: AM, AS, EY (5) drafting the article or revising it critically for important intellectual content: OMGT, AS, EY, AM (3) Final approval of the version to be submitted: OMGT, AS, EY, AM, KK.

All authors read and approved the final manuscript.

Acknowledgement: The authors thank Koç University Research Center for Translational Medicine (KUTTAM), funded by the Republic of Turkey Ministry of Development, for allowing us to use their services and facilities. The content of the manuscript is solely the responsibility of the authors and does not necessarily represent the official views of the Ministry of Development.

This study was presented in part at $2^{\text {nd }}$ International Cell Death Research Congress 2018, Izmir, Turkey, 14 November 2018, and published as congress proceedings MDPI Proceedings 2018,2(25),1552. doi: 10.3390/proceedings 2251552

\section{References}

1. Ramselaar JAM, Boot JP, Van Haeringen NJ, Van Best JA, Oosterhuis JA: Corneal epithelial permeability after instillation of ophthalmic solutions containing local anaesthetics and preservatives. Current eye research 1988, 7(9):947-950.

2. Carney LG, O'Leary DJ, Millodot M: Effect of topical anaesthesia on corneal epithelial fragility. International Ophthalmology 1984, 7(2):71-73. 
3. Pereira VB, Garcia R, Torricelli AA, Bechara SJ: Opioids for Ocular Pain - A Narrative Review. Pain Physician 2017, 20(5):429-436.

4. Benyamin R, Trescot AM, Datta S, Buenaventura R, Adlaka R, Sehgal N, Glaser SE, Vallejo R: Opioid complications and side effects. Pain Physician 2008, 11(2 Suppl):S105-120.

5. Tamura Y, Monden M, Shintani M, Kawai A, Shiomi H: Neuroprotective effects of hibernationregulating substances against low-temperature-induced cell death in cultured hamster hippocampal neurons. Brain Research 2006, 1108(1):107-116.

6. Husain S: Opioid Receptors: Methods for Detection and Their Modes of Actions in the Eye. In: Methods in Molecular Biology. Springer New York; 2014: 243-251.

7. Slaninova J, Appleyard SM, Misicka A, Lipkowski AW, Knapp RJ, Weber SJ, Davis TP, Yamamura HI, Hruby VJ: [125I-Tyr1]biphalin binding to opioid receptors of rat brain and NG108-15 cell membranes. Life Sciences 1998, 62(14):PL199-PL204.

8. Yamazaki M, Suzuki T, Narita M, Lipkowski AW: The opioid peptide analogue biphalin induces less physical dependence than morphine. Life Sciences 2001, 69(9):1023-1028.

9. Sobczak M, Pilarczyk A, Jonakowski M, Jarmuż A, Sałaga M, Lipkowski AW, Fichna J: Antiinflammatory and antinociceptive action of the dimeric enkephalin peptide biphalin in the mouse model of colitis: New potential treatment of abdominal pain associated with inflammatory bowel diseases. Peptides 2014, 60:102-106.

10. Horan PJ, Mattia A, Bilsky EJ, Weber S, Davis TP, Yamamura HI, Malatynska E, Appleyard SM, Slaninova J, Misicka A et al: Antinociceptive profile of biphalin, a dimeric enkephalin analog. $J$ Pharmacol Exp Ther 1993, 265(3):1446-1454.

11. Mollica A, Pinnen F, Costante R, Locatelli M, Stefanucci A, Pieretti S, Davis P, Lai J, Rankin D, Porreca F et al: Biological Active Analogues of the Opioid Peptide Biphalin: Mixed a/ $\beta 3$-Peptides. Journal of Medicinal Chemistry 2013, 56(8):3419-3423.

12. Sahin A, Kam WR, Darabad RR, Topilow K, Sullivan DA. Regulation of leukotriene B4 secretion by human corneal, conjunctival, and meibomian gland epithelial cells. Arch Ophthalmo/ 2012, 130(8):1013-1018.

13. Tian P, Shi W, Liu J, Wang J, Ma C, Qi Q, Cong B, Li Y: Expression of the mu, kappa, and delta-opioid receptors and tyrosine hydroxylase in MN9D cells. International journal of clinical and experimental pathology 2015, 8(5):4863-4868.

14. Bigliardi PL, Neumann C, Teo YL, Pant A, Bigliardi-Qi M: Activation of the $\delta$-opioid receptor promotes cutaneous wound healing by affecting keratinocyte intercellular adhesion and migration. British journal of pharmacology 2014, 172(2):501-514.

15. Wang Y, Gupta M, Poonawala T, Farooqui M, Li Y, Peng F, Rao S, Ansonoff M, Pintar JE, Gupta K: Opioids and opioid receptors orchestrate wound repair. Translational Research 2017, 185:13-23.

16. Gach K, Szemraj J, Stasikowska-Kanicka O, Danilewicz M, Janecka A: Opioid-receptor gene expression and localization in cancer cells. Open Life Sciences 2011, 6(1). 
17. Drago F, Gorgone G, Spina F, Panissidi G, Bello AD, Moro F, Scapagnini U: Opiate receptors in the rabbit iris. Naunyn-Schmiedeberg's Archives of Pharmacology 1980, 315(1):1-4.

18. Drago F, Panissidi G, Bellomio F, Belle AD, Aguglia E, Gorgone G: Effects of opiates and opioids on intraocular pressure of rabbits and humans. Clinical and Experimental Pharmacology and Physiology 1985, 12(2):107-113.

19. Husain S, Abdul Y, Crosson CE: Preservation of Retina Ganglion Cell Function by Morphine in a Chronic Ocular-Hypertensive Rat Model. Investigative Opthalmology \& Visual Science 2012, 53(7):4289.

20. Russell KRM, Wang DR, Potter DE: Modulation of Ocular Hydrodynamics and Iris Function by Bremazocine, a Kappa Opioid Receptor Agonist. Experimental eye research 2000, 70(5):675-682.

21. Stiles J, Honda CN, Krohne SG, Kazacos EA: Effect of topical administration of 1\% morphine sulfate solution on signs of pain and corneal wound healing in dogs. American journal of veterinary research 2003, 64(7):813-818.

22. Peyman GA, Rahimy MH, Fernandes ML: Effects of morphine on corneal sensitivity and epithelial wound healing: implications for topical ophthalmic analgesia. British Journal of Ophthalmology 1994, 78(2):138-141.

23. Lipkowski AW, Konecka AM, Sroczyńska I: Double-enkephalins-Synthesis, activity on guinea-pig ileum, and analgesic effect. Peptides 1982, 3(4):697-700.

24. Kosson D, Klinowiecka A, Kosson P, Bonney I, Carr DB, Mayzner-Zawadzka E, Lipkowski AW: Intrathecal antinociceptive interaction between the NMDA antagonist ketamine and the opioids, morphine and biphalin. European Journal of Pain 2008, 12(5):611-616.

25. Yang L, Shah K, Wang H, Karamyan VT, Abbruscato TJ: Characterization of Neuroprotective Effects of Biphalin, an Opioid Receptor Agonist, in a Model of Focal Brain Ischemia. Journal of Pharmacology and Experimental Therapeutics 2011, 339(2):499-508.

26. Yang L, Islam MR, Karamyan VT, Abbruscato TJ. In vitro and in vivo efficacy of a potent opioid receptor agonist, biphalin, compared to subtype-selective opioid receptor agonists for stroke treatment. Brain Research 2015, 1609:1-11.

27. Chao D, Bazzy-Asaad A, Balboni G, Xia Y. delta-, but not mu-, opioid receptor stabilizes $K(+)$ homeostasis by reducing $\mathrm{Ca}(2+)$ influx in the cortex during acute hypoxia. Journal of Cell Physiology 2007, 212(1):60-67.

28. Lesniak A, Bochynska-Czyz M, Sacharczuk M, Benhye S, Misicka A, Bujalska-Zadrozny M, Lipkowski AW: Biphalin preferentially recruits peripheral opioid receptors to facilitate analgesia in a mouse model of cancer pain - A comparison with morphine. European Journal of Pharmaceutical Sciences 2016, 89:39-49.

29. Popiolek-Barczyk K, Piotrowska A, Makuch W, Mika J: Biphalin, a Dimeric Enkephalin, Alleviates LPSInduced Activation in Rat Primary Microglial Cultures in Opioid Receptor-Dependent and ReceptorIndependent Manners. Neural plasticity 2017, 2017:1-19. 
30. Kawalec M, Kowalczyk JE, Beresewicz M, Lipkowski AW, Zablocka B: Neuroprotective Potential of Biphalin, Multireceptor Opioid Peptide, Against Excitotoxic Injury in Hippocampal Organotypic Culture. Neurochemical Research 2011, 36(11):2091-2095.

31. Mehrotra S, Prajapati RK, Haq W, Singh VK: Immunomodulation by biphalin, dimeric synthetic opioid peptide, and its analog. Immunopharmacology and Immunotoxicology 2002, 24(1):83-96.

32. Zagon IS, Jenkins JB, Sassani JW, Wylie JD, Ruth TB, Fry JL, Lang CM, McLaughlin PJ: Naltrexone, an Opioid Antagonist, Facilitates Reepithelialization of the Cornea in Diabetic Rat. Diabetes 2002, 51(10):3055-3062.

33. Lipkowski AW, Konecka AM, Sroczynska I, Przewlocki R, Stala L, Tam SW: Bivalent opioid peptide analogues with reduced distances between pharmacophores. Life Sciences 1987, 40(23):2283-2288.

34. He X, Sandhu HK, Yang Y, Hua F, Belser N, Kim DH, Xia Y: Neuroprotection against hypoxia/ischemia: 6-opioid receptor-mediated cellular/molecular events. Cellular and Molecular Life Sciences 2012, 70(13):2291-2303.

\section{Titles And Legends For Supplementary Files}

Supplement 1. Video of in vitro scratch assay model showing the effect of $1 \mu \mathrm{M}$ biphalin on relative wound healing area.

Supplement 2. Video of in vitro scratch assay model showing the effect of vehicle on relative wound healing area.

\section{Table}

Table 1. The primers of genes used in the study

\begin{tabular}{ccl}
\hline Gene & Sequence & \\
\hline GAPDH & $\mathrm{F}$ & 5'-ACAACTTTGGTATCGTGGAAGG-3' \\
& $\mathrm{R}$ & 5'-GCCATCACGCCACAGTTTC-3' \\
\hline OPRD1 & $\mathrm{F}$ & 5'-CGTCCGGTACACTAAGATGAAGA-3' \\
& $\mathrm{R}$ & 5'-GCCACGTCTCCATCAGGTA-3' \\
\hline OPRK1 & $\mathrm{F}$ & 5'-ATCATCACGGCGGTCTACTC-3' \\
& $\mathrm{R}$ & 5'ACTCTGAAAGGGCATGGTTGTA-3' \\
\hline OPRM1 & $\mathrm{F}$ & 5'-CAGTGCTCATCATTACCGTGT-3' \\
& $\mathrm{R}$ & 5'-CTGGGATTGTAACCAAGGCTTTA-3' \\
\hline
\end{tabular}


Figures

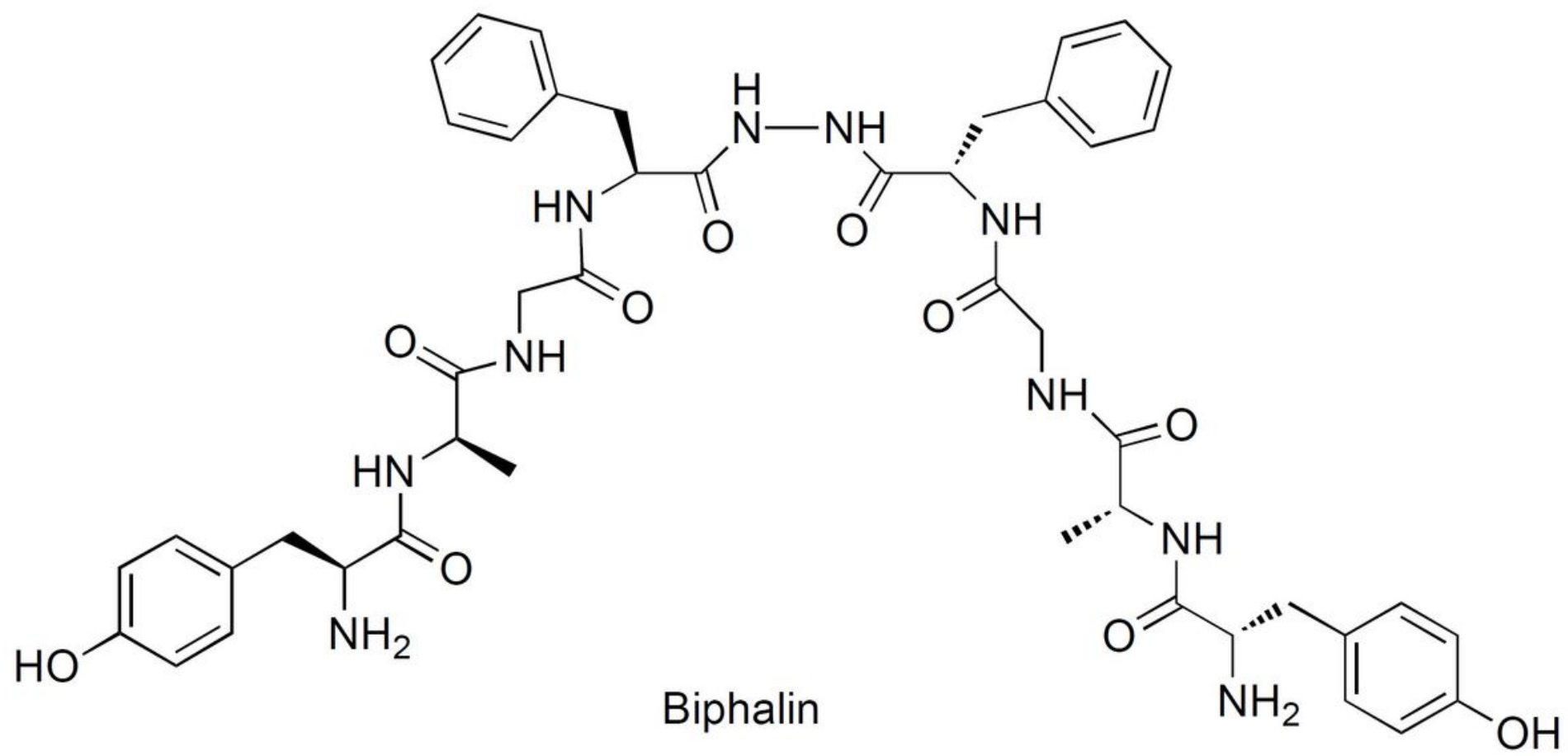

Figure 1

Molecular structure of biphalin. 


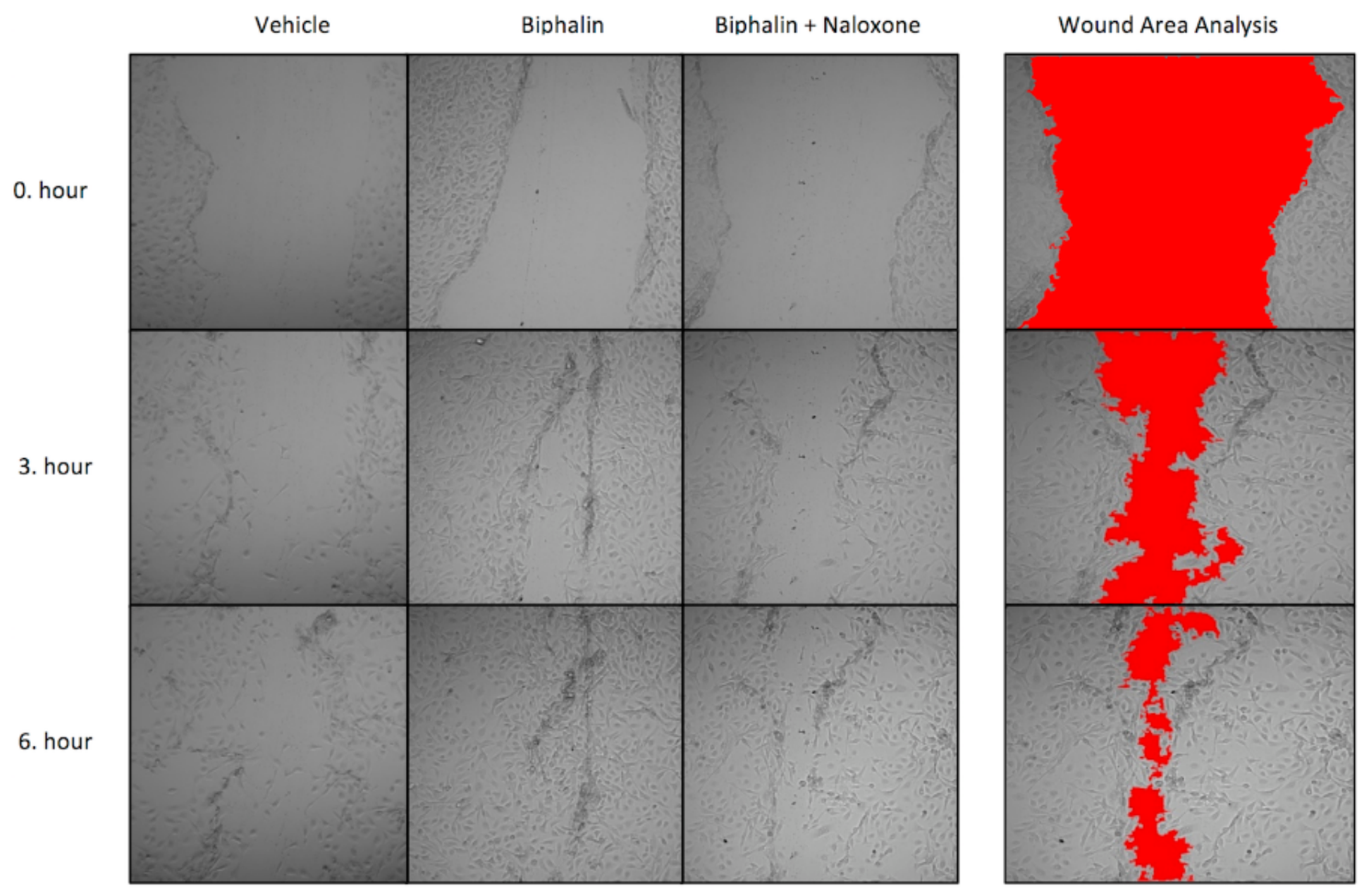

Figure 2

Examples of in vitro live cell migration assays for each experimental group at various time points and example of the relative wound area analysis in ImageJ. 


\section{MTT Toxicity Assay}

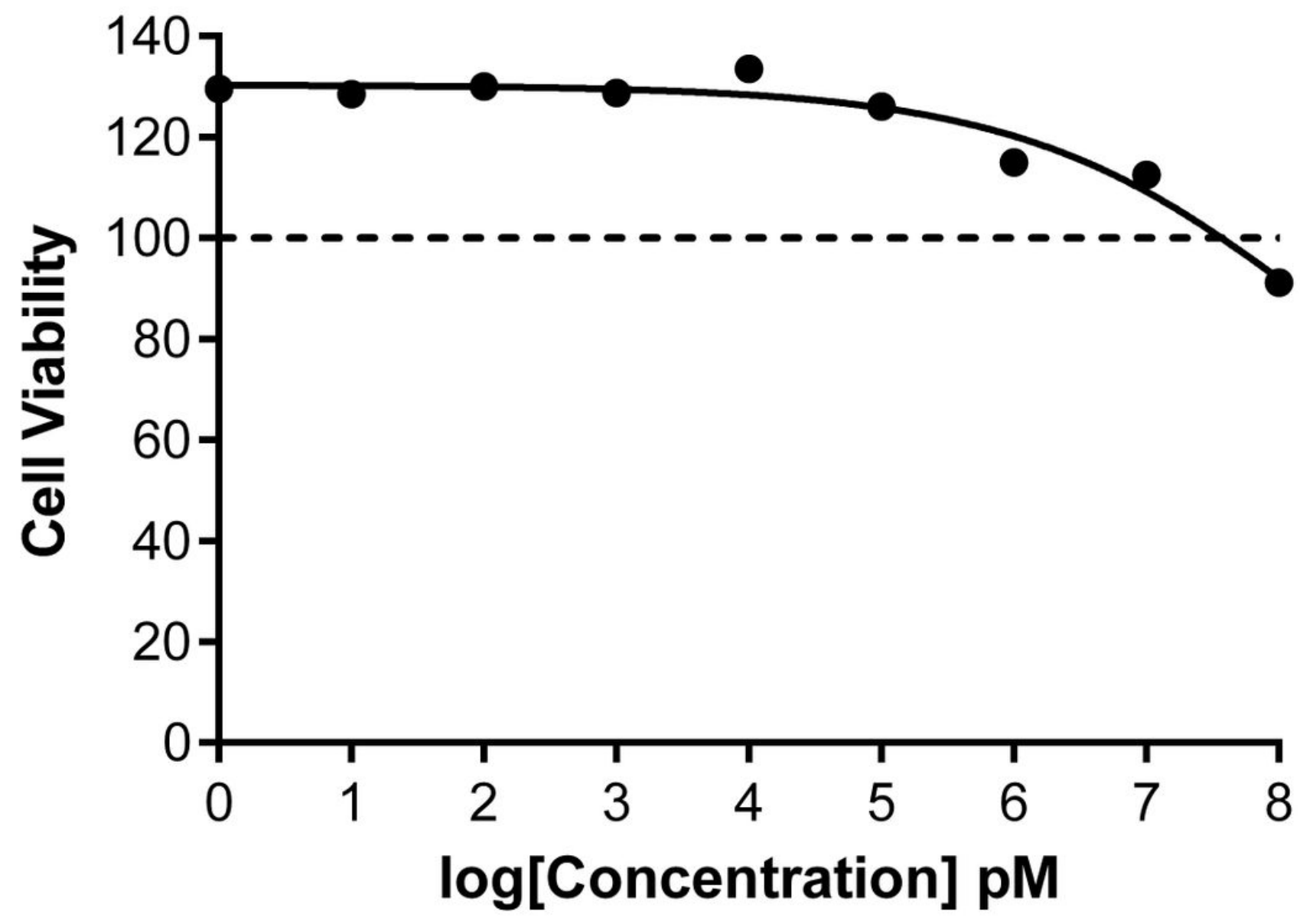

Figure 3

MTT cytotoxicity assay on HCECs for biphalin at various doses after 24-hour of drug incubation $(n=4)$. 
a In Vitro Scratch - $1 \mu \mathrm{M}$

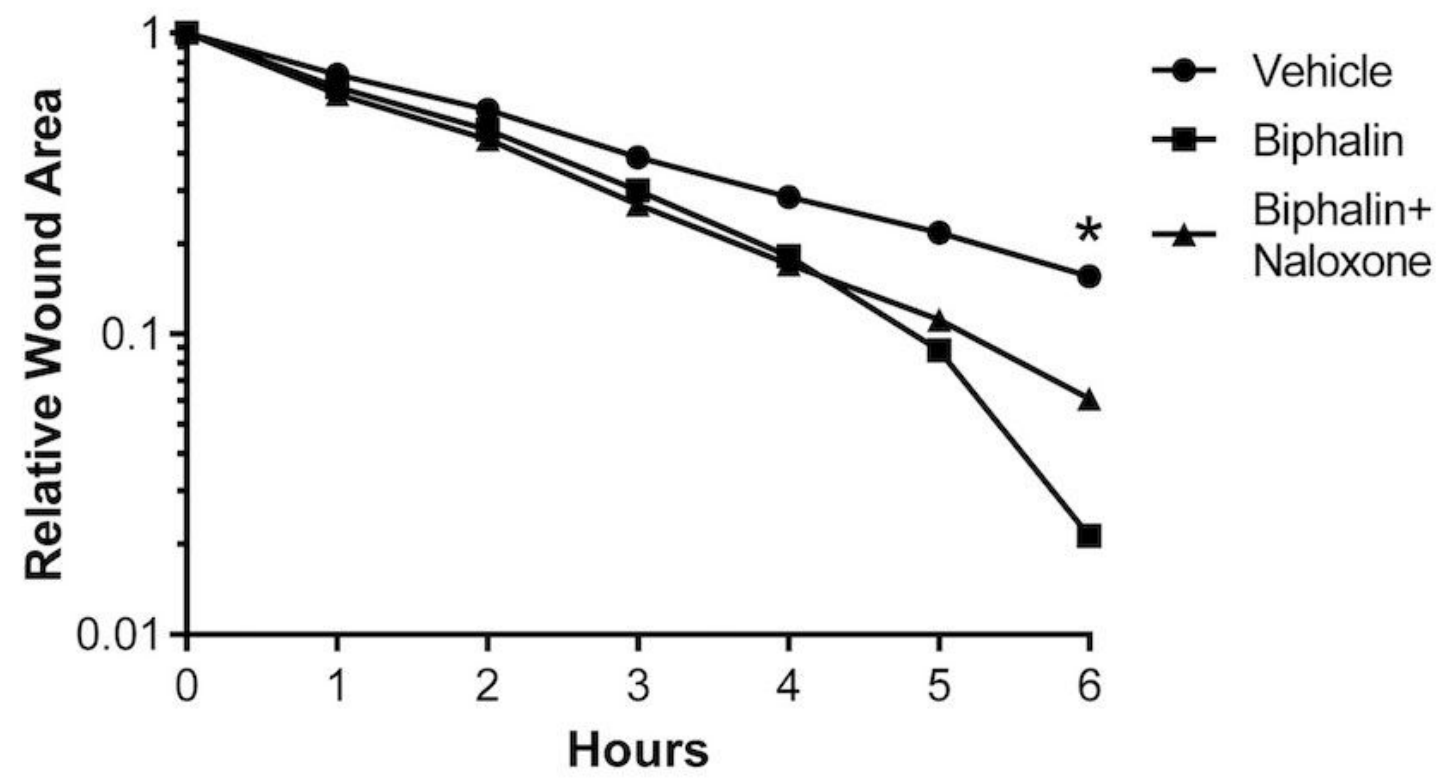

b In Vitro Scratch - $10 \mu \mathrm{M}$

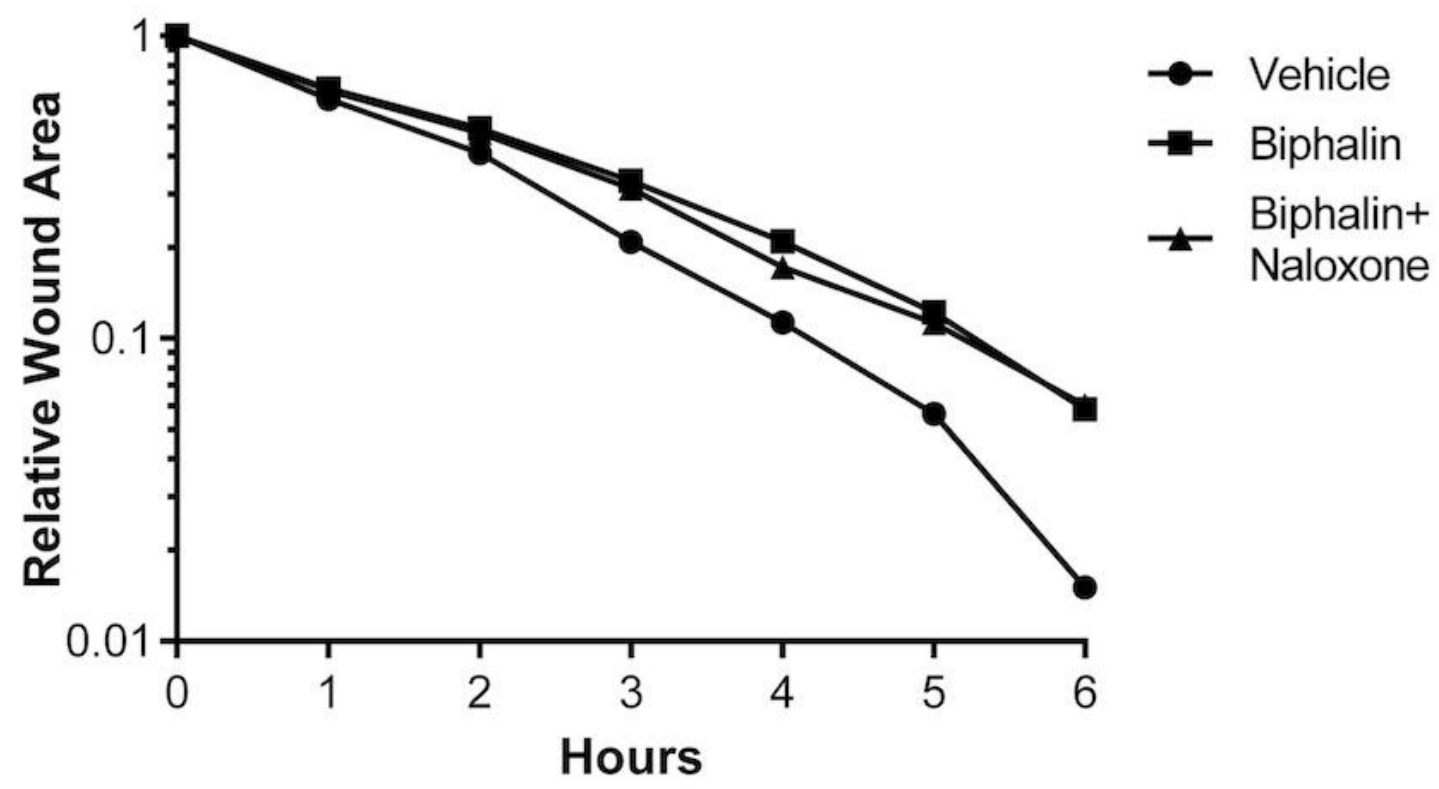

Figure 4

Effects of $1 \mu \mathrm{M}(\mathrm{a})$ and $10 \mu \mathrm{M}$ (b) biphalin and biphalin plus naloxone, and vehicle on in vitro scratch assay model on relative wound healing area (\%). Both of the graphs show the results of two independent experiments $(n=8, * p<0.05)$. 

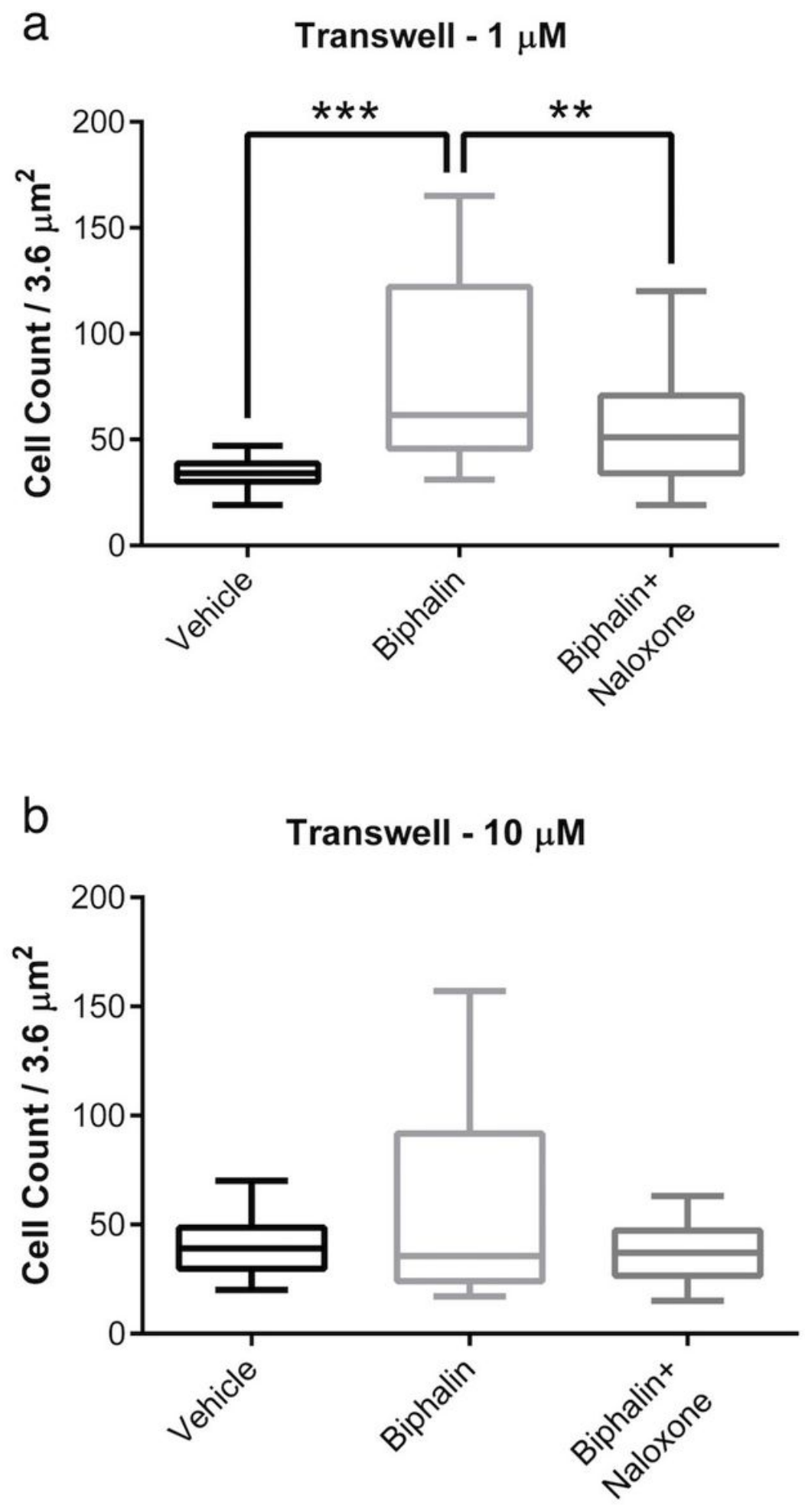

Figure 5

Effects of $1 \mu \mathrm{M}(\mathrm{a})$ and $10 \mu \mathrm{M}(\mathrm{b})$ biphalin and biphalin plus naloxone, and vehicle on the movement of corneal epithelial cells in the transwell migration assay. Both of the graphs show the mean number of migrated cells in ten different micrograph area $(3.6 \mu \mathrm{m} 2)$ in two independent experiments $(n=20, * \star p<$ $\left.0.01,{ }^{\star \star \star *} \mathrm{p}<0.001\right)$. 


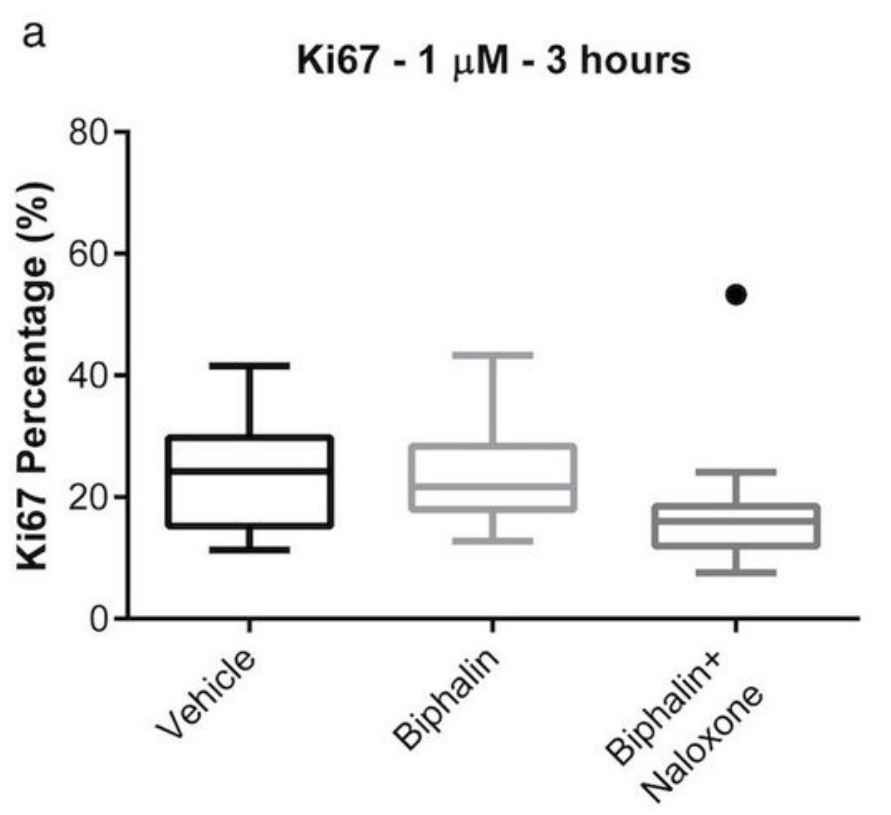

C

Ki67 - $10 \mu \mathrm{M}$ - 3 hours

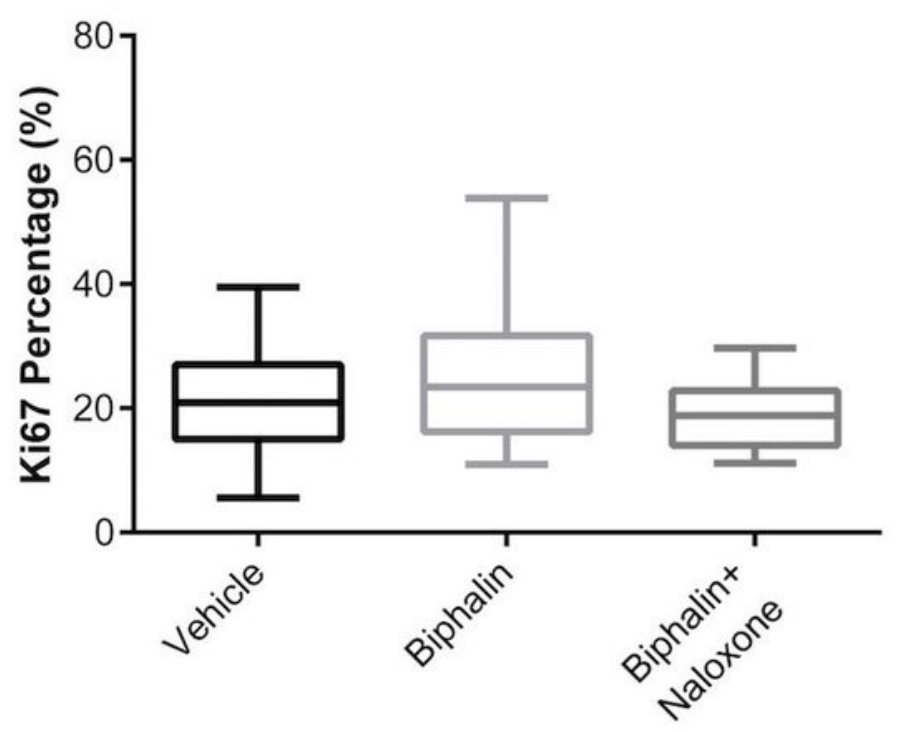

b

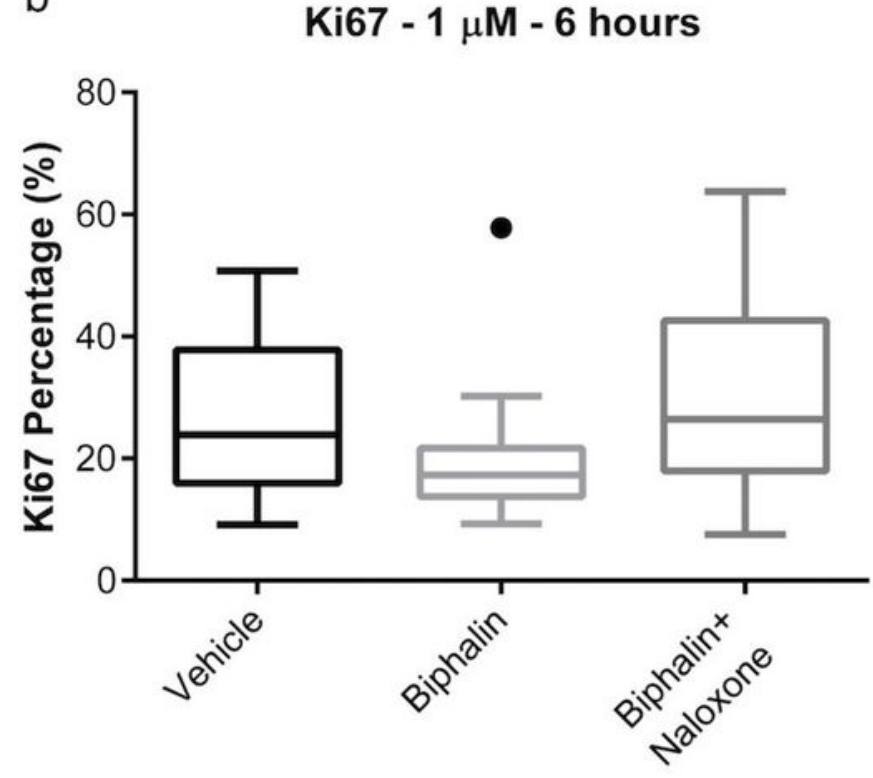

d

Ki67 - $10 \mu M-6$ hours

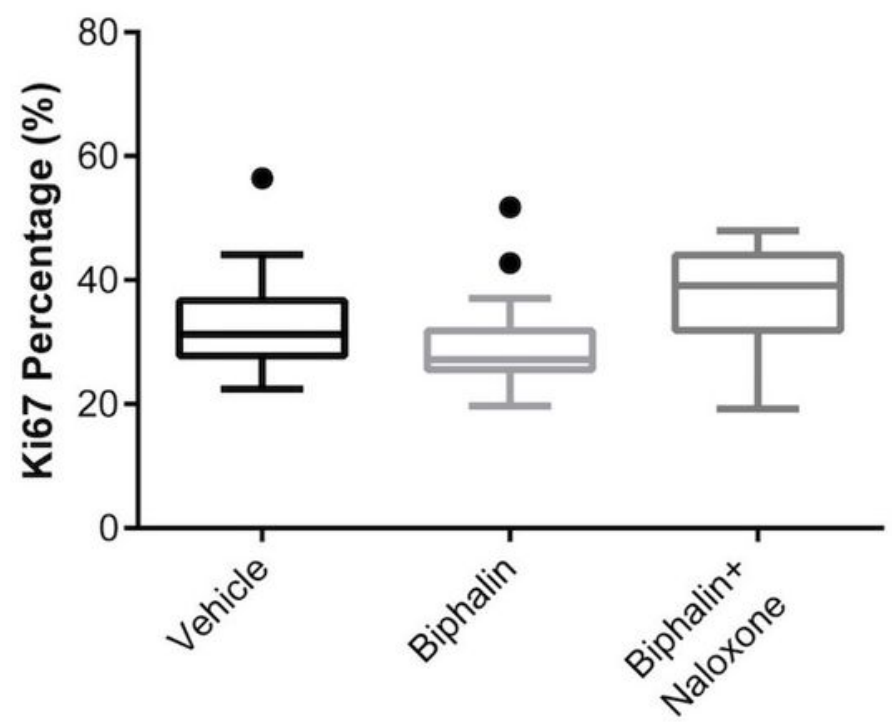

Figure 6

Effects of $1 \mu \mathrm{M}$ biphalin and biphalin plus naloxone, and vehicle at 3 hours (a) and 6 hours (b); and those of $10 \mu \mathrm{M}$ biphalin and biphalin plus naloxone, and vehicle at 3 hours (c) and 6 hours (d) on cell proliferation in Ki67 proliferation assay. The graphs show the percentage of Ki67-labeled cells to DAPIlabeled cells in ten different micrographs in two independent experiments $(n=20)$. 
a
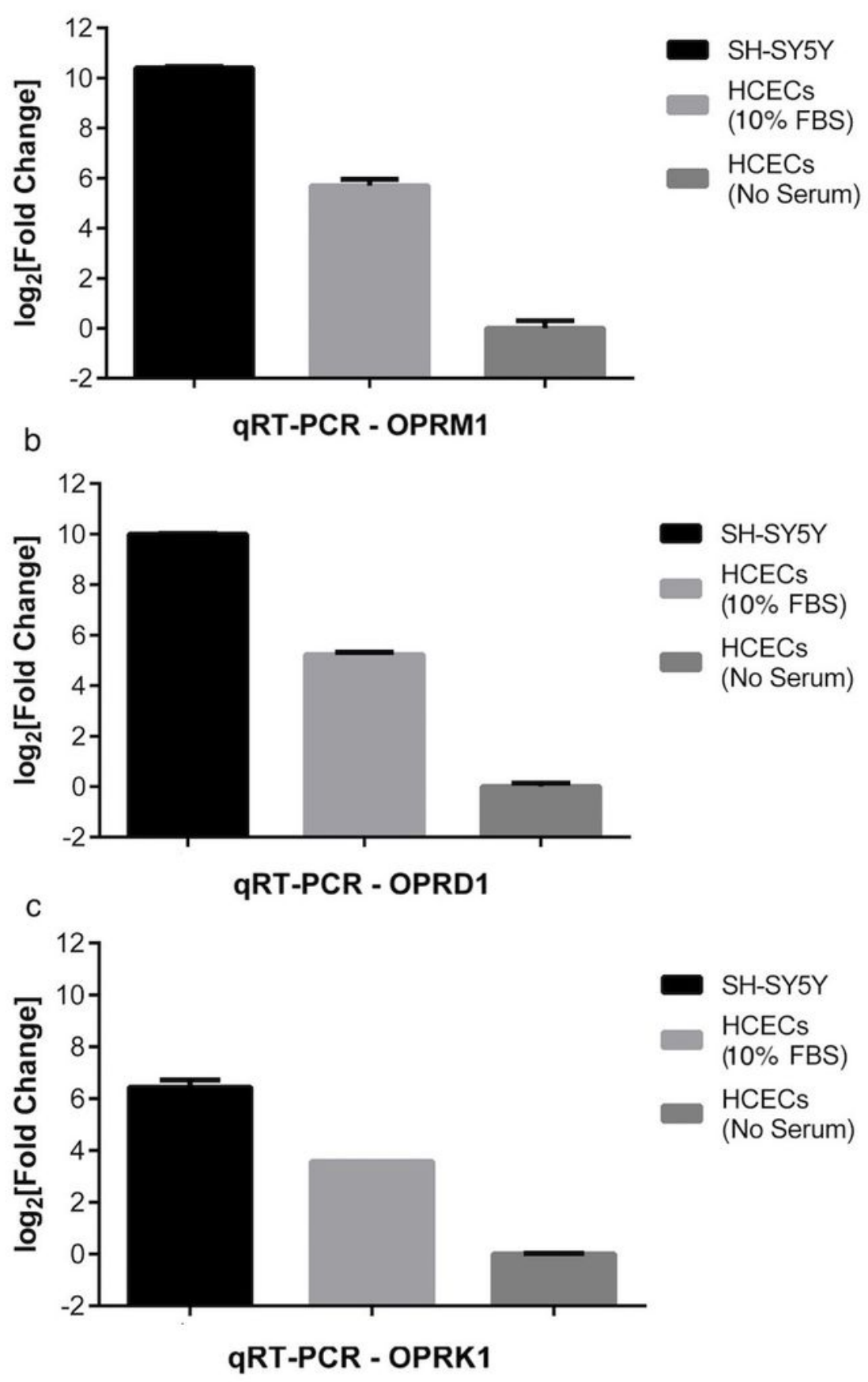

Figure 7

Gene expression analysis with real-time polymerase chain reaction (qRT-PCR) of OPRM1 (a), OPRD1 (b), and OPRK1 (c) mRNAs, which are related with mu, delta and kappa opioid receptor proteins, in HCECs with KSFM or with serum (10\% FBS) and compared with in SH-SY5Y cell lines.

\section{Supplementary Files}


This is a list of supplementary files associated with this preprint. Click to download.

- Supplement1Biphalin.mp4

- Supplement2Vehicle.mp4 